\title{
The Impact of Graft-Versus-Host Disease on Survival in Children with Non-Malignant Disorders Receiving Allogeneic Hematopoietic Cell Transplant
}

\author{
Biljana Horn ${ }^{1}$, Paul Castillo ${ }^{1}$, Mustafa Hanif ${ }^{1}$, Jorge GalvezSilva ${ }^{2}$, Edward Ziga ${ }^{3}$, Warren \\ Alperstein $^{3}$, Micahel Joyce ${ }^{4}$, John Fort ${ }^{1}$, Gauri Sunkerset ${ }^{5}$, Benjamin Oshrine ${ }^{5}$, Jessica \\ Cline $^{1}$, Fan Yang ${ }^{1}$, Jing Zhao ${ }^{1}$, and Deepak Chellapandian ${ }^{5}$ \\ ${ }^{1}$ University of Florida \\ ${ }^{2}$ Nicklaus Children's Hospital \\ ${ }^{3}$ University of Miami Miller School of Medicine \\ ${ }^{4}$ Nemours Children's Health System \\ ${ }^{5}$ Johns Hopkins All Children's Hospital
}

April 9, 2021

\begin{abstract}
Abstract Background: Graft-versus-host disease (GVHD) is a common and undesirable complication of hematopoietic cell transplant (HCT) for non-malignant disorders (NMD). Understanding the incidence and risk factors for GVHD in children with NMD is an important step in developing strategies for its prevention. Study Design: This is a retrospective, registry, study that included children with NMD receiving HCT in 5 centers in Florida between 2010 and 2019. Results: Among 183 patients evaluable for GVHD, acute GVHD (aGVHD) grades I, II, III, and IV were present in 18\%, 12.6\%, 3.8\% and 5.5\% of patients, respectively. Limited and extensive chronic GVHD (cGVHD), were observed in $8.7 \%$ and $12.6 \%$ of patients. Patients with aGVHD grade III/IV had significantly lower 3-year survival rates than those without aGVHD, or those with aGVHD grade I/II (52.9\% [95\% confidence interval (CI) 34-83] vs. 90.1\% [95\% CI 84-96], vs. 98.1\% [95\%CI 95-100], p<0.001). Patients without cGVHD and those with limited and extensive cGVHD had 3-year survival rates of $88.9 \%$ [95\%CI 84-94], 91.7\% [95\%CI 77-100], and $84.8 \%$ [95\%CI 70-100], respectively, log rank $\mathrm{p}=0.3$. Receiving transplant from an HLA-mismatched unrelated donor (MMUD), as compared to a matched related donor (MRD), increased the risk for aGVHD grade III/IV (Odds ratio 10.4 [95\% CI 2.5-47.6]). There were no cases of aGVHD grade III/IV among recipients of mismatched related/haploidentical transplants. Conclusions: Grade III/IV aGVHD, which significantly reduced overall survival, was reported in $9.3 \%$ of children with NMD receiving HCT. Risk factors included HCT from a MMUD but not mismatched related donors.
\end{abstract}

The Impact of Graft-Versus-Host Disease on Survival in Children with Non-Malignant Disorders Receiving Allogeneic Hematopoietic Cell Transplant

Biljana Horn, MD ${ }^{1}$, Paul Castillo, MD ${ }^{1}$, Mustafa Hanif ${ }^{1}$, Jorge Galvez Silva, MD ${ }^{3}$, Edward Ziga, $\mathrm{MD}^{4}$, Warren Alperstein, $\mathrm{MD}^{4}$, Michael Joyce, $\mathrm{MD}, \mathrm{PhD}^{5}$, John Fort, $\mathrm{MD}^{1}$, Gauri Sunkersett, $\mathrm{DO}^{2}$, Benjamin Oshrine, MD², Jessica Cline, BS ${ }^{1}$, Fan Yang, MS ${ }^{1}$, Jing Zhao, PhD.MS ${ }^{1}$ and Deepak Chellapandian, MD $\mathrm{MBBS}^{2}$

Affiliations:

(1) University of Florida, Gainesville, FL 
(2) Center for Cell and Gene Therapy for Non Malignant-Conditions, Blood and Marrow Transplant Program, Cancer \& Blood Disorders Institute, Johns Hopkins All Children's Hospital, St Petersburg, FL; (3) Nicklaus Children's Hospital, Miami, FL

(4) Pediatric Hematology and Oncology, University of Miami School of Medicine, Miami, FL (5) Nemours Children's Clinic and Wolfson Children's Hospital, Jacksonville, FL

Corresponding Author:

Email: Biljana Horn; e-mail: Biljana.horn@ufl.edu,

1600 SW Archer Avenue, Gainesville, FL 32610

Phone (mobile) 415-215-6930, FAX:352 2948091

Article Type: Research Article

Word Count: abstract 246

Word Count: main text 2571

Tables: 3, Figures: 2, References: 21, Supporting files: 0

Short Title: GVHD in Children with Non-Malignant Disorders

Key Words: GVHD, pediatric HCT, non-malignant disorders, Florida consortium

Abbreviations used in the manuscript:

\begin{tabular}{ll}
\hline ATG & Anti-thymocyte globulin \\
\hline BM & Bone Marrow \\
CIBMTR & Center for International Blood and Marrow Transplant Research \\
FPBCC & Florida Pediatric Bone Marrow Transplant and Cell Therapy Consortium \\
GVHD, aGVHD, cGVHD & Graft-versus-host disease, acute graft versus-host disease, chronic graft-versus-host disease \\
HCT & Hematopoietic cell transplant \\
HLA & Human leukocyte antigen \\
MMUD & Mismatched unrelated donor \\
MMRD & Mismatched related donor \\
MRD & Matched related donor \\
MUD & Matched unrelated donor \\
NMD & Non-malignant disorders \\
OR & Odds ratio \\
OS & Overall survival \\
PB & Peripheral blood \\
UCB & Umbilical cord blood \\
\hline
\end{tabular}

Previous Presentations: Abstract presentation during the virtual Transplantation and Cellular Therapy Meeting, February 8-12, 2021 under title: Risk of Clinically Significant Graft-Versus-Host Disease in Children Receiving Allogeneic Hematopoietic Cell Transplant (HCT) for Non-Malignant Disorders in Florida (20102019).

\section{Abstract \\ Background:}

Graft-versus-host disease (GVHD) is a common and undesirable complication of hematopoietic cell transplant (HCT) for non-malignant disorders (NMD). Understanding the incidence and risk factors for GVHD in children with NMD is an important step in developing strategies for its prevention. 


\section{Study Design:}

This is a retrospective, registry, study that included children with NMD receiving HCT in 5 centers in Florida between 2010 and 2019.

\section{Results:}

Among 183 patients evaluable for GVHD, acute GVHD (aGVHD) grades I, II, III, and IV were present in $18 \%, 12.6 \%, 3.8 \%$ and $5.5 \%$ of patients, respectively. Limited and extensive chronic GVHD (cGVHD), were observed in $8.7 \%$ and $12.6 \%$ of patients. Patients with aGVHD grade III/IV had significantly lower 3 -year survival rates than those without aGVHD, or those with aGVHD grade I/II (52.9\% [95\% confidence interval (CI) 34-83] vs. 90.1\% [95\% CI 84-96], vs. 98.1\% [95\%CI 95-100], p<0.001). Patients without cGVHD and those with limited and extensive cGVHD had 3-year survival rates of $88.9 \%$ [95\%CI 84-94], 91.7\% [95\%CI 77-100], and 84.8\% [95\%CI 70-100], respectively, log rank $p=0.3$. Receiving transplant from an HLA-mismatched unrelated donor (MMUD), as compared to a matched related donor (MRD), increased the risk for aGVHD grade III/IV (Odds ratio 10.4 [95\% CI 2.5-47.6]). There were no cases of aGVHD grade III/IV among recipients of mismatched related/haploidentical transplants.

\section{Conclusions:}

Grade III/IV aGVHD, which significantly reduced overall survival, was reported in $9.3 \%$ of children with NMD receiving HCT. Risk factors included HCT from a MMUD but not mismatched related donors.

\section{Introduction}

Allogeneic hematopoietic cell transplantation is used to treat a variety of malignant and non-malignant hematologic disorders. (1,2) In patients with NMD, rejection, treatment-related mortality, and GVHD are the main obstacles to favorable outcomes.

Graft-versus-host disease is an immunologically mediated process involving donor immune cell responses against major or minor histocompatibility antigens of the recipient. Acute GVHD is driven by donor's lymphocytes and inflammatory cytokine cascade, while chronic GVHD involves inflammation, immune dysregulation and fibrosis. $(3,4)$ In children with malignant disorders, graft-versus-host effect may correlate with graft-versus-leukemia effect and contribute to reduction of relapse; $(5,6)$ however, there is no benefit of GVHD in patients with NMD, and any degree of GVHD in this population is considered an undesirable iatrogenic effect. Understanding the incidence and risk factors for GVHD in children with NMD is in important step in developing strategies for its prevention.

In this report we describe the incidence of acute and chronic GVHD in a contemporary cohort of children receiving HCT for NMD, describe survival outcomes based on GVHD grades, and identify risk factors for aGVHD grade III/IV, which correlated with inferior survival.

\section{Study Design}

For this retrospective, registry study, we gathered data from the Florida Pediatric Bone Marrow Transplant and Cell Therapy Consortium (FPBCC). The objectives of FPBCC are to identify pediatric HCT best practices and improve survival of children receiving HCT in Florida. FBPCC participation involves monthly virtual meetings, participation in quality improvement projects, sharing data for retrospective trials, and developing consortium-wide prospective clinical trials. FPBCC was founded in 2018 and comprises 5 of the 6 pediatric transplant programs in the state of Florida, USA. All FPBCC participating centers signed memoranda of understanding and data use agreements and obtained institution-specific IRB approvals for this retrospective data analysis. All patients have signed the informed consent for data reporting. Transplant centers report detailed data on consecutive hematopoietic cell transplantations to the statistical center of the Center for International Blood and Marrow Transplant Research (CIBMTR), and compliance with this reporting is monitored by on-site audits. Data from participating centers were downloaded from the enhanced data back to center platform of CIBMTR and forwarded to the FPBCC statistical center at the University of Florida (Gainesville, FL, USA), where data were combined into a single set and analyzed. 
We describe characteristics of pediatric HCT recipients, including gender, age, race, performance score, diagnosis, type of transplant, and number of pre-transplant comorbidities. Transplant characteristics consist of type of donor, stem cell source, Human Leukocyte Antigen (HLA) match, conditioning regimen, and regimen intensity. Outcomes data include length of survival, cause of death, and incidence and grade of GVHD. Our data set included information on timing and use of second transplant; however, reasoning (e.g. early or late graft rejection) for second transplant was not available and the dataset did not contain details of donor lymphocyte infusions or details of GVHD prophylaxis other than the use of serotherapy. Thus patients who required the $2^{\text {nd }}$ or subsequent transplant $(\mathrm{N}=21)$ and those who rejected the transplant but did not undergo a subsequent transplant $(\mathrm{N}=3)$ were not included in this manuscript. Patients for whom the center did not provide information on GVHD $(\mathrm{N}=3)$ were considered not-evaluable for GVHD and excluded as well. Myeloablative regimens were defined as those using one or more of the following: total body irradiation $>500$ cGy for a single dose or $>800$ cGy for fractionate, busulfan $>7.2 \mathrm{mg} / \mathrm{kg}$ iv, melphalan $>150 \mathrm{mg} / \mathrm{m}^{2}$, or thiotepa [?]10 mg/kg. Reduced intensity regimens were defined as those using melphalan [?]150 mg/m², thiotepa $<10 \mathrm{mg} / \mathrm{kg}$, TBI $>200 \mathrm{cGy}$ and [?]500 cGy as a single dose or [?] 800 cGy fractionated, busulfan [?]7.2 mg/kg. Non-myeloablative regimens used any dose of ATG, fludarabine, cyclophosphamide, or TBI[?]200 cGy. (7) Fully HLA-matched unrelated bone marrow (BM) or peripheral blood (PB) donors were matched at A, B, C, and DRB1 antigens by high-resolution typing. For umbilical cord blood (UCB) donors, full match was defined as 6/6 HLA-antigen match (A, B, DRB1) by either low or high resolution. A related donor was considered haploidentical if [?] 2 different antigens (A, B, C, or DRB1) were mismatched. CIBMTR gathers and reports the highest grade of acute GVHD by one year post transplant following criteria published by Przepiorka et al. (8) Although currently CIBMTR gathers data on individual organ involvement with cGVHD based on NIH Consensus Criteria, 2014, the data available through our platform contained only information on extent of cGVHD by one year post transplant.

cGVHD was reported as limited or extensive based on definition by Shulman et al. (9) Limited cGVHD includes only localized skin involvement and/or liver dysfunction, while any other organ involvement is considered extensive.

\section{Statistical analyses}

Descriptive statistics were used for summarizing patient and transplant characteristics. Overall survival (OS) was defined as the duration of time from transplant to time of death or the date of the last follow-up reported to the CIBMTR database. The Kaplan-Meier method was used to estimate overall survival and the log-rank test was used to examine the effect of acute and chronic GVHD on overall survival. Odds ratio (OR) analysis was used to identify factors associated with the development of aGVHD grade III/IV. Analyses were performed with SPSS@ statistical program version 25-26 (IBM Corp., Armonk, N.Y., USA) and R Core Team, 2020.

\section{Results}

\section{Patient characteristics}

Table 1 Patient and transplant characteristics describes details of 183 children undergoing first allogeneic HCT for NMD in Florida during 2010-2019, who had data on GVHD reported to CIBMTR. The most common diagnoses included sickle cell disease $(24.0 \%)$, severe aplastic anemia $(21.9 \%)$, primary immunodeficiencies including severe combined immunodeficiency (24.1\%), thalassemia (11.5\%) and bone-marrow failure syndromes $(8.2 \%)$. Median age of children was 7 years (range $0-21$ ) with even distribution among $<3$ years, 3-9 years, and [?]10 years of age groups. Graft types included bone marrow (BM), umbilical cord blood (UCB), and peripheral blood (PB) used in $69.9 \%, 18.6 \%$, and $9.8 \%$ of patients, respectively. Donors were related in $53.6 \%$ of cases and unrelated in the rest. Fully HLA-matched donors were used in $65.6 \%$ of HCT, mismatched in $31.1 \%$, and for $3.3 \%$ of patients the degree of match was unknown. Conditioning regimens were myeloablative in $55.2 \%$ of patients, and $4.4 \%$ of patients did not receive any conditioning, while all other patients received non-myeloablative or reduced intensity regimens. Low dose (200cGy) total body irradiation (TBI) was used in $7.6 \%$ of $\mathrm{HCT}$ recipients. Anti-thymocyte globulin was used in conditioning 
of $55.2 \%$ of patients and alemtuzumab in $34.4 \%$. Rituximab was used as part of GVHD prophylaxis by one center for a majority of their transplant recipients, and thus $23.0 \%$ of patients received a single dose of rituximab during conditioning.

Survival for the entire cohort and causes of death

Kaplan-Meier estimated overall survival for the entire cohort at 36 months was $88.5 \%$ [95\% CI 84-94]. Twenty-three (12.6\%) patients have died, with bacterial infections being responsible for $7(30.4 \%)$ deaths, fungal infections for 1 (4.3\%), organ failures for $8(34.8 \%)$, recurrence of underlying disease for $3(13.0 \%)$, secondary malignancy for 1 (4.3\%), while acute and chronic GVHD were responsible for 2 deaths (8.6\%). For 1 patients $(4.3 \%)$, the cause of death was unknown.

Incidence of GVHD and survival based on GVHD grade

Out of 183 evaluable patients, $110(60.1 \%)$ did not have any evidence of aGVHD, 33 (18\%) had aGVHD grade I, $23(12.6 \%)$ grade II, $7(3.8 \%)$ grade III, and $10(5.5 \%)$ grade IV. One hundred forty-four patients $(78.7 \%)$ did not have cGVHD, 16 (8.7\%) had chronic limited, and 23 (12.6\%) had chronic extensive GVHD. Table 2 describes the incidence of GVHD by donor relation, stem cell source and HLA-match. Patients receiving unrelated mismatched donor BM, PB, or mismatched UCB had the highest incidence of aGVHD $68.7 \%$ and $63.2 \%$, respectively). The incidence of aGVHD in haploidentical and MRD recipients was $36.4 \%$ and $29.6 \%$, respectively. However, there was no aGVHD grade III/IV among haploidentical HCT recipients. FIGURE 1 depicts overall survival by acute GVHD grade and FIGURE 2 depicts overall survival by cGVHD grade. Patients without aGVHD, aGVHD grade I/II, and aGVHD grade III/IV had 3-year survival of 90.1\% [95\%CI 84-96], 98.1\% [95\%CI 95-100], and 52.9\% [95\%CI 34-83], respectively. The difference in survival among the 3 groups was significant $(\log$ rank $\mathrm{p}<0.0001)$. Patients without cGVHD and those with limited and extensive cGVHD had 3-year survival rates of $88.9 \%$ [95\%CI 84-94], 91.7\% [95\%CI 77-100], and 84.8\% [95\%CI 70100], respectively. Neither limited nor extensive cGVHD significantly affected 3-year survival rates (log rank $\mathrm{p}=0.3)$.

Risk factors for development of grade III/ IV aGVHD

Because aGVHD grade III/IV adversely affected survival, we analyzed risk factors for its development as shown in TABLE 3 Risk factors for developing aGVHD grade III/IV. The risk of aGVHD grade III/IV was higher in patients receiving HLA mismatched donor transplant (OR 3.4 [95\% CI 1.1-11.2]. However, not all recipients of mismatched donor transplants were at risk of aGVHD grade III/IV. Only the recipients of mismatched unrelated donor HCTs comparing to MRD had an increased risk of grade III/IV aGVHD (OR 10.4 [95\% CI 2.5-47.6]). Among recipients of [?]2 Ag mismatched related donor transplants, there were no cases of aGVHD grade III/IV. The use of mismatched cord blood donors was not associated with slight increase in risk of aGVHD grade III/IV in comparison with MRD (OR 1.3 [95\% CI 0.1-8.0]).

Causes of death in patients with and without aGVHD grade III/IV

At a mean follow-up, for alive patients, of 38.9 months (+-SD 25.8 months) 15/166 (9\%) and 8/17 (47\%) of patients without and with aGVHD grade III/IV were deceased, respectively. The causes of death in patients without and with aGVHD grade III/IV included recurrent disease (13.3\% vs $12.5 \%$ ), organ failure (40\% vs $25 \%$ ), bacterial infection (33\% vs $25 \%$ ), fungal infection ( 0 vs $12.5 \%$ ) GVHD (0\% vs $25 \%$ ), $2^{\text {nd }}$ malignancy (6.7\% vs $0 \%)$, and unknown $(6.7 \%$ vs $0 \%)$.

\section{Discussion}

We describe acute and cGVHD incidence in a contemporary cohort of children with NMD undergoing allogenic HCT. There is abundant literature on the incidence and risk factors for GVHD in adult and pediatric HCT recipients with malignant disorders; however, similar reports are scarce for children with NMD. The larger published studies describing impact of GVHD on clinical outcomes included patients transplanted in the 1990s (5) or included patients transplanted over several decades. $(10,11)$ Given changes in HCT practices, it was important to validate these results on a contemporary cohort of patients. 
Regarding incidence of GVHD in pediatric patients with NMD, our findings are similar to findings from the Japanese transplant registry, which included all children with NMD transplanted between 1985 and 2016. (10) Umeda et al. reported cumulative incidences of aGVHD grade II-IV, aGVHD grade III-IV, and cGVHD of $24 \%, 9.1 \%$ and $17.8 \%$, compared with $21.9 \%, 9.3 \%$ and $21.3 \%$ in our cohort. (10)

Our data shows that aGVHD grade III/IV, which developed in $9.3 \%$ of children with NMD, significantly affected the overall survival, while aGVHD grade I/II has no adverse impact on survival. Due to small numbers of deceased patients, we could not identify significant differences in cause of death between patients with and without aGVHD grade III/IV. The risk of infections was similar between the 2 groups and GVHD was considered the primary cause of death in 2 patients. Similar to previous reports, we could not document significantly reduced survival rates in children with cGVHD $(5,10)$. Even when it does not affect survival, due to related morbidity and impact on quality of life, any acute or cGVHD represents an unwanted iatrogenic complication in children with NMD.

Due to genetic diversity of Florida's population, finding a fully HLA-matched donor is difficult and $31 \%$ of our patients received stem cells from alternative donors such as mismatched cord blood, mismatched unrelated donors, and mismatched related donors. Our study indicates that only recipients of mismatched unrelated BM and PB had an increased risk of aGVHD grade III/IV, which was related to reduced survival. The incidence of aGVHD grade III/IV was lower in mismatched related donor recipients than in MRD, and was similar in mismatched UCB recipients to MRD.

Previous publications (Bertaina A et al., Zecca et al., Anurathapan et al.) reported absence of aGVHD grade III/IV in haploidentical transplant recipients, regardless which of the 3 depletion strategies was used (CD34 positive selection, $\alpha \beta$ T-cell and CD19+ cell depletion, or in vivo T-cell depletion with post-transplant cyclophosphamide). (13-15)

In our previous analysis of data from the FPBCC, we observed inferior survival of children with NMD receiving HCT from mismatched unrelated donors, including mismatched cord blood, and we recommended that use of mismatched related donors be favored over mismatched unrelated BM, PB or UCB donors. (16) Findings from this study indicate that improved methods of GVHD prophylaxis are needed in children with NMD receiving mismatched unrelated BM or PB. Recent literature describes use of abatacept, alpha beta ( $\alpha \beta)$ T-cell and CD19-cell depletion, and post-transplant cyclophosphamide as promising methods for GVHD prophylaxis in children with NMD. (13,17-19) However, donor selection and the approach to GVHD prophylaxis when an HLA-matched donor is lacking, is the subject of research and until large randomized studies are available, the alternative donor selection and GVHD prophylaxis will likely remain center-specific. Only 1/5 FPBCC transplant centers performed ex-vivo T-cell depletion/CD34+ selection during the study period, and no centers performed $\alpha \beta$ T-cell depletion, while all centers used post-transplant cyclophosphamide, which was used in the majority of haploidentical HCT recipients in this study. Post-transplant cyclophosphamide use results in excellent outcomes and significant reduction in grade III/IV GVHD; however, higher risk of graft failure in pediatric non-malignant conditions and hemoglobinopathies, and post-transplant late effects related to high dose chemotherapy warrant caution when using this method of GVHD prophylaxis in children with NMD. $(20,21)$ Literature indicates that alpha beta CD3+/CD19+ cell depleted haploidentical HCT resulted in excellent survival, low GVHD rates, and good immune reconstitution, and this method is emerging as a promising strategy for GVHD prevention in children with NMD. (13) However, some investigators feel that significant myeloablation, required for T-cell depleted transplants, is a disadvantage of this approach.

Due to its retrospective, registry nature, our study lacked some relevant data, such as agents used for GVHD prophylaxis or method of T-cell depletion. Although we included 10-year pediatric transplant data, the numbers, particularly in some subgroups, are still low, emphasizing that transplants for pediatric NMD are rare events (only 20-25 of these transplants are done annually in Florida) and that consolidating data, not only across centers but across different NMD diagnostic groups, is necessary in order to understand outcomes and complications of those transplants.

\section{Conclusions}


Acute GVHD grade III/IV developed in 9.3\% of children undergoing HCT for NMD, and it was related to an adverse 3-year overall survival (52.9\% [95\% CI 34-83]). Although contributing to morbidity, grade I/II aGVHD or cGVHD did not impact overall survival. Recipients of mismatched unrelated BM or PB transplants, but not those of mismatched related donor transplants or mismatched cord blood transplants, were at a high risk for aGVHD grade III/IV. In order to reduce the incidence of aGVHD grade III/IV and improve survival, we recommend either avoiding mismatched unrelated donors or using novel methods for GVHD prophylaxis, ideally done on prospective clinical trials.

\section{Conflict of Interest Statement}

The authors of this manuscript declare that they do not have relevant conflicts of interest in the subject matter discussed.

\section{Acknowledgement}

We gratefully acknowledge the support of Dr. Douglas Rizzo, Senior Scientific Director from CIBMTR who introduced us to the eDBtC platform. We thank the nonprofit Children's Miracle Network for their 2018 and 2020 grants in support of FPBCC.

\section{References}

1. Ljungman P, Bregni M, Brune M, Cornelissen J, De Witte T, Dini G et al. Allogeneic and autologous transplantation for haematologic diseases, solid tumours and immune disorders: current practice in Europe 2009. Bone Marrow Transplant. 2010;45(2):219-234.

2. Passweg J, Baldomero H, Bader P, Bonini C, Cesaro S, Dreger P, et al. The EBMT activity survey report 2017: a focus on allogeneic HCT for nonmalignant indications and on the use of non-HCT cell therapies. Bone Marrow Transplant. 2019;54:1575-1585.

3. Gooptu M, Koreth J. Translational and clinical advances in acute graft-versus-host disease. Haematologica 2020 Nov 1;105(11):2550-2560. doi:10.3324/haematol.2019.240309.

4. MacDonald K, Hill G, Blazer B. Chronic graft-versus-host disease: biological insights from preclinical and clinical studies. Blood 2017;129:13-21.

5. Zecca M, Prete A, Rondelli R, Lanino E, Balduzzi A, Messina C et al. Chronic graft-versus-host disease in children: incidence, risk factors, and impact on outcome. Blood. 2002;100:1192-1200.

6. Pulsipher M, Langholz B, Wall D, Schultz K, Bunin N, Carroll W, et al. The addition of sirolimus to tacrolimus/methotrexate GVHD prophylaxis in children with ALL: a phase 3 Children's Oncology/Pediatric Blood and Marrow Transplant Consortium trial. Blood.2014 Mar 27;123(13):2017-25.

7. Bacigalupo A, Ballen K, Rizzo D, Giralt S, Lazarus H, Ho V, et al. Defining the intensity of conditioning regimens: Working Definitions. Biol Blood Marrow Transplant 2009; 15:1628-33.

8. Przepiorka D, Weisdorf D, Martin R, Klingemann HG, Beatty P, Hows J, et al. 1994 Consensus conference on Acute GVHD Grading. Bone Marrow Transplant.1995;15:825-8.

9. Shulman HM, Sullivan KM, Weiden PL, McDonald GB, Striker GE, Sale GE, et al. Chronic graftversus-host syndrome in man. A long-term clinicopathologic study of 20 Seattle patients. Am J Med.1980;69:204-17.

10. Umeda K, Imai K, Yanagimachi M, Yabe H, Kobayashi M, Takahashi Y, et al. Impact of graftversus-host disease on the clinical outcome of allogeneic hematopoietic stem cell transplantation for non-malignant diseases. International Journal of Hematology 2020;111:869-876.

11. Mahmoud H, Elhaddad A, Fahmy O, Samra M, Abdelfattah R, El-Nahass Y, et al. Allogeneic hematopoietic stem cell transplantation for non-malignant hematological disorders. Journal of Advanced Research. 2015;6:449-438.

12. Jacobsohn D, Arora M, Klein J, Hassebroek A, Flowers M, Cutler C et al. Risk factors associated with increased nonrelapse mortality and with poor overall survival in children with chronic graft-versus-host disease. Blood. 2011;118:4472-4479.

13. Bertaina M, Merli P, Rutella S, Pagliara D, Bernardo ME, Masetti R, et al. HLA-haploidentical stem cell transplantation after removal of alphabeta- $\mathrm{T}$ and $\mathrm{B}$ cells in children with non-malignant disorders. 
Blood. 2014;124(5):822-6.

14. Zecca M, Strocchio L, Pagliara D, Comoli P, Bertaina A, Giorgiani G, et al. HLA-haploidentical Tcell depleted allogeneic hematopoietic stem cell transplantation in children with Fanconi anemia. Biol Blood Marrow Transplant. 2014;20(4):571-6.

15. Anurathapan U, Hongeng S, Pakakasama S, Sirachainan N, Songdej D, Chuansumrit A, et al. Hematopoietic stem cell transplantation for homozygous beta-thalassemia and betathalassemia/hemoglobin E patients from haploidentical donors. Bone Marrow Transplant. 2016;51(6):813-8.

16. Chellapandian D, Sunkersett G, Oshrine B, Galvez Silva J, Ziga E, Alperstein W, et al. Pediatric HCT in Florida (2014-2016): A Report from the FPBCC. Pediatric Transplant. 2020 Nov 27;313931. Doi: 10.1111/petr.13931Horan J, Wang T, Haagenson M, Spellman S, Dehn J, Eapen M, et al. Evaluation of HLA matching in unrelated hematopoietic stem cell transplantation for non-malignant disorders. Blood. 2012;120:2918-2924.

17. Ngwube A, Shah N, Jacobsohn D, Ziga D, Shenoy S. Abatacept is effective for Gvhd prophylaxis after unrelated donor stem cell transplantation (URD SCT) for severe sickle cell disease. Blood 2019134 (Supplement_1:370). https://doi.org/10.1182/blood-2019-126405.

18. Bertaina A, Pitisci A, Sinibaldi M, Algeri M. T Cell-depleted and T cell-replete HLA-Haploidentical stem cell transplantation for non-malignant disorders. Curr Hematol Malig Rep. 2017;12:68-78.

19. Arcuri L, Nabhan S, Cunha R, Nichele S, Ribeiro A, Fernandes J, et al. Impact of CD34 cell dose and conditioning regimen on outcomes after haploidentical donor hematopoietic stem cell transplantation with post-transplantation cyclophosphamide for relapsed/refractory severe aplastic anemia. Biol Blood Marrow Transplant. 2020;26:2311-2317.

20. Even-Or E, NaserEddin A, Schejter YD, Shadur B, Zaidman I and Stepensky P. Bone Marrow Transplant. 2020. https://doi.org/10.1038/s41409-020-01040-9.

21. Yu J, Black V, Lamba J, Horn B. Potential risk factors associated with graft failure of haploidentical hematopoietic stem cell transplantation (HSCT) in Children with sickle cell disease (SCD). J Pediatr Hematol Oncol. 2020 Jun 26. doi:10.1097/MPH.0000000000001873. Figure Legends: FIGURE 1 Overall survival by acute GVHD grade FIGURE 2 Overall survival by chronic GVHD grade Data: The data that support the findings of this study are available from the corresponding author upon a reasonable request. .

\section{Hosted file}

Table 1 GVHD.pdf available at https://authorea.com/users/406895/articles/517377-the-impactof-graft-versus-host-disease-on-survival-in-children-with-non-malignant-disordersreceiving-allogeneic-hematopoietic-cell-transplant

\section{Hosted file}

TABLE 2 Incidence of GVHD by donor relation.pdf available at https://authorea.com/users/ 406895/articles/517377-the-impact-of-graft-versus-host-disease-on-survival-in-childrenwith-non-malignant-disorders-receiving-allogeneic-hematopoietic-cell-transplant

\section{Hosted file}

Update TABLE 3 Risk factors for developing aGVHD grade III.pdf available at https://authorea. com/users/406895/articles/517377-the-impact-of-graft-versus-host-disease-on-survival-inchildren-with-non-malignant-disorders-receiving-allogeneic-hematopoietic-cell-transplant 


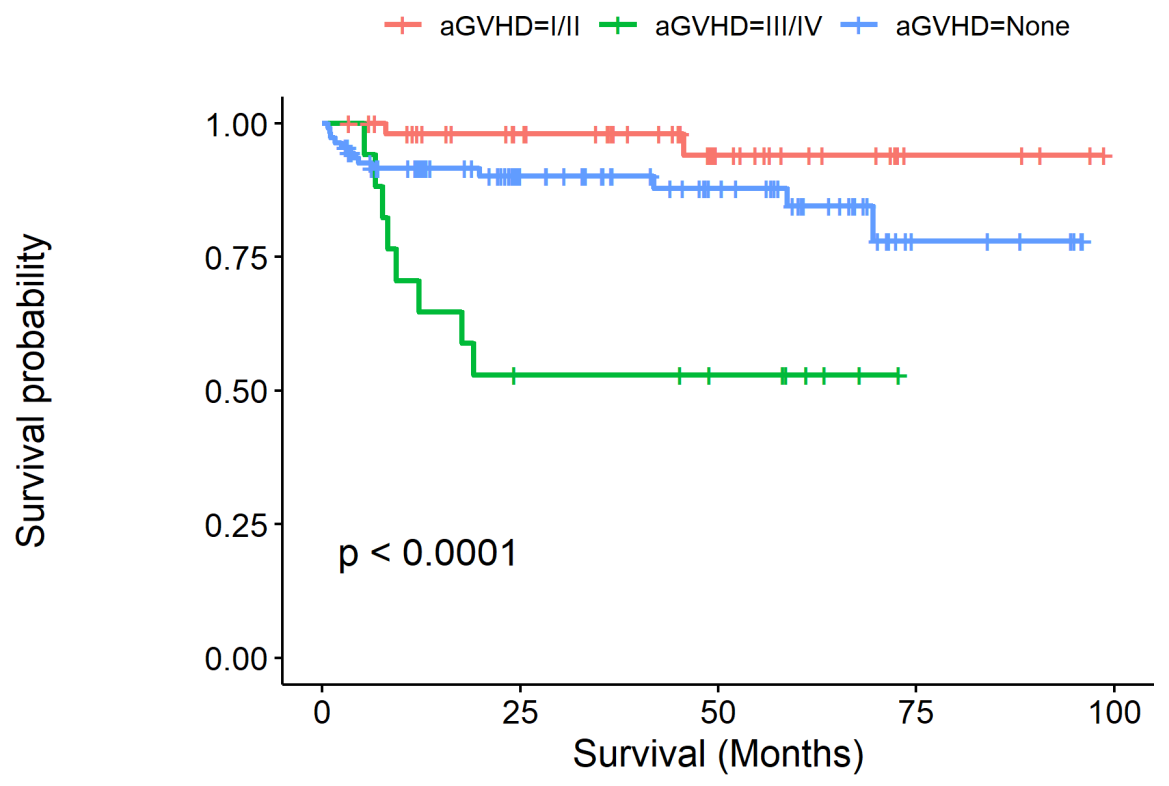

\begin{tabular}{|c|c|c|c|c|c|}
\hline \multicolumn{6}{|c|}{ Number at risk } \\
\hline $\begin{array}{r}a G V H D=I / I I- \\
a G V H D=I I / / I V \\
a G V H D=\text { None }\end{array}$ & $\begin{array}{l}56 \\
17 \\
110\end{array}$ & $\begin{array}{c}39 \\
8 \\
51\end{array}$ & $\begin{array}{c}18 \\
6 \\
33\end{array}$ & $\begin{array}{l}4 \\
0 \\
6\end{array}$ & $\begin{array}{l}0 \\
0 \\
0\end{array}$ \\
\hline & 0 & 25 & $\begin{array}{r}50 \\
\text { al }(\mathrm{N}\end{array}$ & 75 & 100 \\
\hline
\end{tabular}


$+c G V H D=$ None $+c G V H D=$ Limited $+c G V H D=$ Extensive

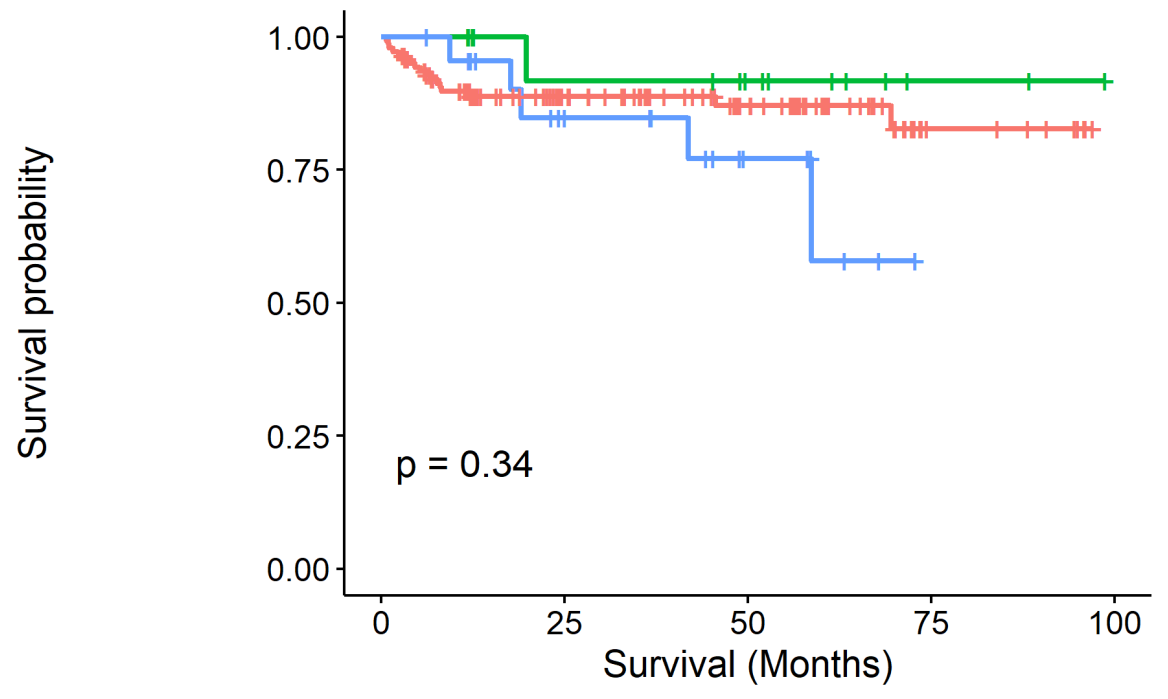

Number at risk

\begin{tabular}{|c|c|c|c|c|c|}
\hline $\begin{array}{r}\text { cGVHD= None } \\
\text { cGVHD=Limited } \\
\text { cGVHD=Extensive }\end{array}$ & $\begin{array}{c}144 \\
16 \\
23 \\
\end{array}$ & $\begin{array}{l}74 \\
11 \\
13\end{array}$ & $\begin{array}{c}43 \\
8 \\
6 \\
\end{array}$ & $\begin{array}{l}8 \\
2 \\
0\end{array}$ & $\begin{array}{l}0 \\
0 \\
0\end{array}$ \\
\hline & 0 & 25 & 50 & 75 & 100 \\
\hline
\end{tabular}

